\title{
Analysis of Natural Laminar Flow Aircraft based on Airline Network Design and Fleet Assignment
}

\author{
Matthias Braun, ${ }^{*}$ Kai Wicke* and Alexander Koch* \\ German Aerospace Center (DLR), Hamburg, 21079, Germany \\ Tobias Wunderlich ${ }^{\dagger}$ \\ German Aerospace Center (DLR), Brunswick, 38108, Germany
}

\begin{abstract}
This paper demonstrates the evaluation of a future aircraft concept equipped with natural laminar flow technology (NLF) based on airline network design and fleet assignment. NLF leads to higher fuel efficiency compared to existing state-of-the-art short-to-medium haul aircraft. The evaluation approach based on network modeling allows to capture network effects such as fleet utilization. Network effects are not captured by a cost benefit analysis applied to standard flight missions. In this study networks are designed for different air transportation scenarios, whereas the flight schedule of an existing European low-cost carrier serves as candidate flight list. Fleet assignment is solved simultaneously by considering a baseline aircraft and the future aircraft concept as candidate fleets. The resulting networks are evaluated with respect to global network profitability, fuel consumption and fleet composition. Results reveal a significant impact of the natural laminar flow performance on fleet deployment and network contribution of the future concept. Further, results indicate turn-around time to be an important driver for fleet deployment caused by network effects.
\end{abstract}

\section{Nomenclature}

$\begin{array}{ll}A S K & \text { available seat kilometer } \\ B E L F & \text { break-even load factor } \\ B S W & \text { backward swept wing } \\ c_{L} & \text { lift coefficient } \\ C F M U & \text { central flow management unit } \\ C F R P & \text { carbon fibre reinforced plastic } \\ D O C & \text { direct operating cost } \\ F S W & \text { forward swept wing } \\ \text { ICA } & \text { initial cruise altitude } \\ M T O W & \text { maximum take-off weight } \\ N L F & \text { natural laminar flow } \\ O E W & \text { operating empty weight } \\ \operatorname{PrADO} & \text { preliminary aircraft design and optimization program }\end{array}$

\section{Introduction}

Laminar flow is a promising future aircraft technology improvement with the potential to increase aircraft fuel efficiency and to reduce the climate impact of aviation. ${ }^{1}$ Laminar flow technology decreases the aircraft drag by increasing the regions with a laminar boundary layer e.g., on wing surfaces. Consequently, the aircraft

*Research Engineer, Air Transportation Systems, Blohmstr. 18.

${ }^{\dagger}$ Research Engineer, Institute of Aerodynamics and Flow Technology, Lilienthalplatz 7. 
requires less fuel for the same mission than current state-of-the-art aircraft. However, the technology exhibits some drawbacks which might limit its future operability. For example, laminar flow condition leading to the drag advantage is unlikely to be preserved in all flight phases. That is, laminar flow is approximately limited to cruise flight conditions. ${ }^{2}$ In addition, the turn-around time of laminar flow aircraft might increase e.g., due to a change of the aircraft configuration or increased maintenance effort, which affects aircraft utilization in network operations. From an airline perspective, the network benefits of this future technology concept are of particular interest. Network benefits include e.g., changes in airline network profit or network fuel consumption due to the introduction of laminar flow aircraft in network operations. The objective of this paper is an operational analysis of the natural laminar flow (NLF) concept in terms of network benefits achievable by a single airline compared to a state-of-the-art baseline fleet.

Methodology for the operational analysis is based on airline network modeling. In particular, a network design model with integrated fleet assignment is used to design cost-effective airline networks for different air transportation scenarios. Cost-effectiveness in this context refers to global airline network profit. Scenarios capture variations in fuel price, aircraft turn-around time and achievable laminar flow area. A single airline network is designed for each scenario considered, whereas the flight schedule of an existing European low-cost carrier serves as a candidate flight list. Further, fleet assignment is solved simultaneously on the network while accounting for fleet utilization. The resulting networks and fleet assignment solutions are compared with a baseline network designed with a conventional short-to-medium haul aircraft fleet. The approach allows for a qualitative and quantitative analysis of the network benefits achievable by a single airline by introduction of the future concept into its operational fleet. Computation of block fuel rates for different flight distances are based on flight mission simulations with a flight trajectory model. All flight mission simulations are carried out in a preliminary step to the network analysis. The resulting block fuel regressions dependent on flight distance serve as input for network design and calculation of direct operating costs for all candidate flights in the network.

Some basic assumptions are set for this study: First, this analysis focuses on natural laminar flow in short-to-medium haul operations, i.e., on network operations with aircraft similar to the A320 or the Boeing 737. The application of laminar flow technology in the short-to-medium range is a worst case scenario as the fuel efficiency potential is best exploited on long-haul routes. However, from an airline perspective, shortto-medium haul operation is a dominating part in the air transportation system in terms of seat kilometers flown. In addition, laminar flow technology may be a potential candidate for implementation in an A320 successor, supported by rising fuel prices and the challenge to reduce aviation's climate impact. Second, this study is based on two preliminary aircraft designs developed at DLR. The first design represents a state-of-the-art short-to-medium haul aircraft similar to the A320. This first aircraft design defines the baseline fleet for network modeling. The second design is based on the first one but equipped with NLF. This results in a changed aircraft configuration compared to the baseline aircraft such as the use of forward swept wings (FSW). Third, natural laminar flow technology is limited to the wings of the aircraft only. That is, optimum laminar flow in this study refers to maximum laminar flow condition at the wings only, representing about $15 \%$ of the aircraft's surface. Finally, no additional maintenance costs for the NLF-fleet compared to the baseline fleet are assumed. However, the turn-around time of the NLF-fleet is increased in this study compared to the baseline aircraft design. Increased turn-around time is expected due to the changed configuration of the NLF-aircraft or increased maintenance effort e.g., a cleaning of the wings after each flight.

Organization of this paper is as follows: Section II reviews the natural laminar flow concept and the two aircraft designs. Section III introduces the network design and fleet assignment model forming the core part of the methodology. Experiment design including calculation of block fuel rates is outlined in Section IV. Network results are presented in Section V with a conclusion in Section VI.

\section{Natural Laminar Flow Aircraft Concept}

This section introduces the two aircraft designs given as input for this operational study. Both aircraft concepts were designed at the DLR Institute of Aerodynamics and Flow Technology with the preliminary aircraft design software PrADO ${ }^{\mathrm{a}}$. An overview of the design procedures of PrADO can be found in $3,4$. Two different types of aircraft were designed and serve as candidate fleet for network design. The concept of operation of both fleet types is listed in table 1. The first aircraft design represents a current state-of-the-art

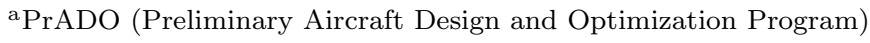


short-to-medium haul configuration (see figure 1) with turbulent wing design similar to the current A320 family. This type of aircraft represents the baseline fleet.

Table 1. Concept of operation FSW-NLF aircraft.

\begin{tabular}{ll} 
Requirement & Value \\
\hline \hline Range & $4,800 \mathrm{~km}$ \\
Payload & $14,250 \mathrm{~kg}$ \\
& $(150 \mathrm{Pax}$ incl. baggage $)$ \\
Cruise speed & $0.78 \mathrm{Ma}$ \\
Maximum take-off field length & $7,000 \mathrm{ft}$ \\
Maximum landing field length & $6,500 \mathrm{ft}$
\end{tabular}
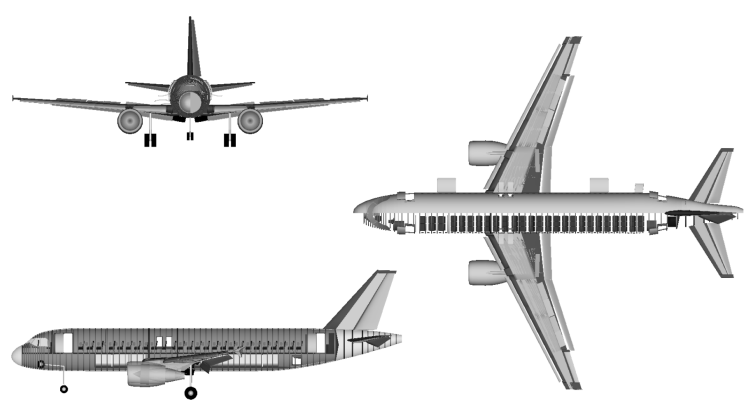

Figure 1. Three side view baseline aircraft.

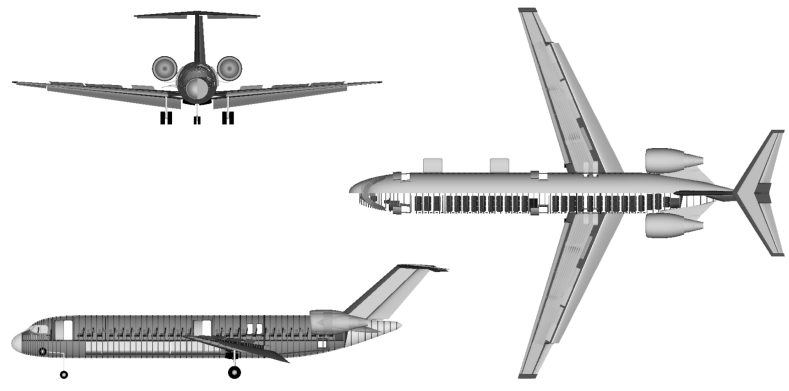

Figure 2. Three side view FSW-NLF configuration.

The second FSW-NLF aircraft design is also based on an A320 type of aircraft and additionally equipped with NLF at the wings (see figure 2). The operation of an aircraft with NLF technology results in a reduced aircraft drag, implicating lower fuel consumption and less carbon dioxide emissions. Natural laminar flow is a passive technology improvement, i.e., no further systems need to be installed. Looking at the aircraft components, the wing is particularly applicable for NLF. However, designing a wing with NLF becomes a trade-off between different contradictory requirements. In order to consider transonic effects and to maintain the cruise speed of today's transport aircraft, a certain wing sweep is required. With respect to the application of laminar flow, the design of a swept wing is constrained by three primary transition mechanisms, namely transition caused by Tollmien-Schlichting instabilities and crossflow instabilities as well as attachment line transition. ${ }^{6-8}$

To prevent boundary-layer instability effects at high Reynolds numbers, the leading edge sweep angle for a standard medium sized aircraft equipped with NLF is limited to values around $10-15^{\circ} .5$ This limitation would result in an undesirable reduction of cruise speed. Using a FSW instead of a backward swept wing (BSW) implies some advantages for the application of NLF as well as for transonic design. The FSW has a reduced leading edge sweep angle creating a more stable laminar boundary layer with respect to crossflow and attachment line transition. However, the relevant sweep for transonic aerodynamics (sweep of $50 \%$ chord line) remains the same and thus the transonic characteristics of the FSW are comparable to the BSW. ${ }^{7,9}$ The main disadvantage of forward wing sweep design is the tendency for aeroelastic divergence causing higher wing bending moments e.g., due to gust loads. ${ }^{8}$ This is normally counteracted by an increased stiffness of the wing structure, causing higher wing weights. ${ }^{7}$ Approaching this problem, carbon fibre reinforced plastic (CFRP) can be used for aeroelastic tailoring were the deformational behavior of the wing is systematically affected. ${ }^{10,11}$ In combination with the material properties of CFRP the weight penalty can be lessened. Another requirement for applying NLF is a very high surface quality with respect to surface roughness and waviness. ${ }^{2,6}$ The current aluminum manufacturing techniques with its rivets and surface discontinuities are not sufficient for realizing adequate surface qualities for NLF. Manufacturing wings with CFRP materials may provide a solution for this problem.

In this study, laminar flow is maintained over the forward part of the wing by an appropriate airfoil design. An exemplary distribution of the transition location during cruise for the FSW-NLF configuration 
is plotted in figure 3. In the outer wing area, a maximum transition location of $55 \%$ local wing depth was assumed. The inner wing area is characterized by higher Reynolds numbers which cause a transition location closer to the leading edge.

Figure 2 shows that the aircraft is equipped with a T-tail and rear-mounted engines, in order to create a clean wing and to achieve optimum laminar flow conditions. The T-tail configuration would also allow to install new engine concepts with very high bypass ratio, like geared turbofans or propfans. Looking at the leading edge high lift system, the aircraft has smart leading edge devices ${ }^{12}$ instead of conventional slats, providing a stepless and gapless wing surface.

Finally, the performance data of the two aircraft types in table 2 reveal a higher operating empty weight (OEW) of the FSW-NLF aircraft as a result of the T-tail configuration and the structural disadvantage of the forward swept wing. Even though the wing is made of CFRP, the weight penalty of the structural reinforcements can only be compensated partially. However, the maximum take-off weight (MTOW) is only $0.6 \%$ higher, justified by the lower required mission fuel on the design mission. Based on the better aerodynamical performance, the engines are sized with less thrust.

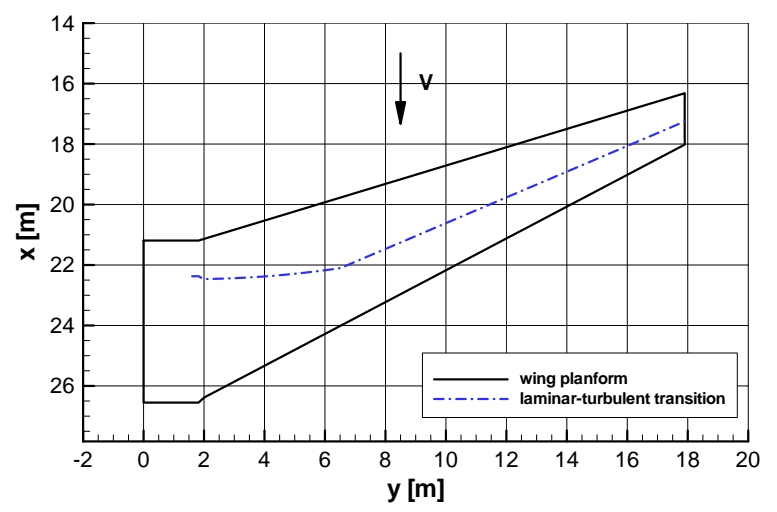

Figure 3. Exemplary computation of the laminar-turbulent transition location during cruise.

Table 2. Configuration characteristics of the two aircraft types used as candidate fleet for network design.

\begin{tabular}{lccc} 
Parameter & Baseline aircraft & FSW-NLF Aircraft & $\Delta$ \\
\hline \hline MTOW $[k g]$ & 72,550 & 73,000 & $+0.6 \%$ \\
OEW $[k g]$ & 41,350 & 43,600 & $+5.4 \%$ \\
Sea level static thrust $[k N]$ & 111.1 & 107.6 & $-3.2 \%$ \\
Cruise L/D [-] & 16.43 & 19.56 & $+19.1 \%$
\end{tabular}

\section{Concept Evaluation Based on Airline Network Modeling}

The network impact of the NLF concept compared to conventional short-to-medium haul aircraft is analyzed with an airline network design and fleet assignment model. This model has been developed in the context of this work. Airline schedule planning is a complex decision making process with the aim to produce a minimum cost flight schedule while adhering to a variety of operational constraints such as airport slot availability, allocation of resources like fleet and crew to flights in a network, and many others. The planning of a flight schedule is a large-scale optimization task. So far, the planning task cannot be solved at once but is separated into a set of sequential planning stages, each of them representing a more simple subproblem. Those subproblems usually include network design, fleet assignment, aircraft maintenance routing and crew planning. An introduction to airline schedule planning models is given by Gopalan and Talluri. ${ }^{13}$ In particular, a standard formulation of the fleet assignment problem is provided by Hane et. al. ${ }^{14}$ An integrated network design and fleet assignment model was proposed by Lohatepanont et.al., ${ }^{15}$ who formulated an incremental network design approach based on an existing base schedule. The model used in 
this work serves as a systems analysis tool for the evaluation of the future NLF aircraft concept in comparison to an existing baseline fleet. The network design and fleet assignment model (NEMO) is positioned at a strategic level in order to support the analysis of future air transportation system concepts while capturing network effects such as turn-around times or connecting traffic. This is an advantage compared to a simple cost-benefit analysis approach applied to standard flight missions.

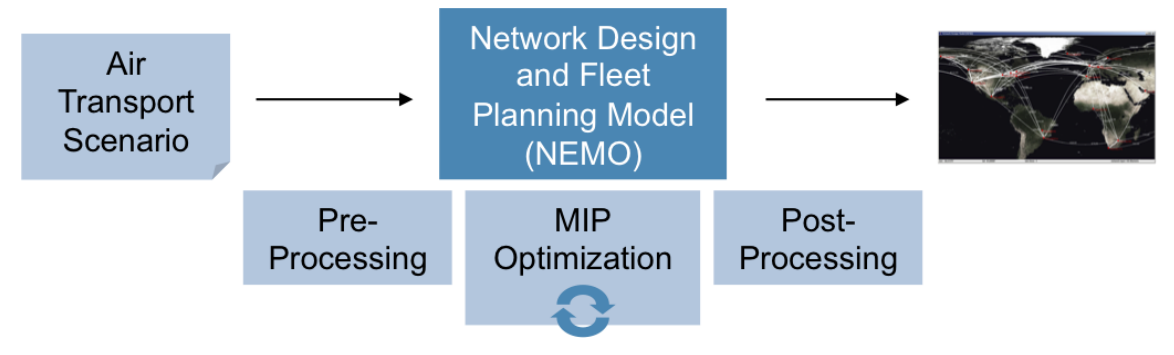

Figure 4. Airline network design model with integrated fleet assignment.

Given an air transportation scenario characterized by a set of city-pairs with associated passenger demand, ticket prices, operating costs and a candidate fleet for network operations, the optimization model designs a cost-effective airline network from scratch (see figure 4). That is, the airline network is designed from the bottom up rather than a modification of an existing flight schedule. Here, cost-effectiveness refers to global network profit defined as global network revenue less global direct operating costs. Network design from scratch is achieved by first constructing a candidate flight list serving as a network design space in the optimization process. This candidate flight list is constructed in a pre-processing step in advance to the optimization. However, in order to study a specific airline case, an existing airline flight schedule can be used as a candidate flight list, providing a fleet assignment solution for the given air transportation scenario. The optimizer selects flights from the candidate flight list to construct a network such that global network profit is maximized and all operational constraints are satisfied. Constraints include fleet assignment constraints such as aircraft availability, flight coverage and aircraft flow balance. Further constraints are passenger demand, aircraft seat capacity and turn-around time constraints. In addition, airport slot constraints are considered. The result is a fleeted flight schedule (network) at a strategic level that is analyzed in a post-processing step with respect to its performance in terms of costs, profit, fuel consumption and others.

In particular, network design decisions include all operated flights with departure and arrival times, the routing of passengers in the network and the operational fleet. Operational fleet is the type and number of aircraft used for network operation including fleet assignment decisions. Fleet assignment assigns a particular fleet type to each flight in the network while considering constraints such as aircraft flow balance and aircraft availability. In addition, fleet capital costs are considered for each aircraft in operation. The network design model is formulated as a mixed integer program (MIP). Network design and fleet decisions are solved simultaneously based on an integrated model formulation. The planning horizon of the model is set to a single day.

\section{Experiment Design}

\section{A. Network Design Assumptions}

Three air transportation scenarios capturing variations in fuel price, natural laminar flow condition and turn-around time are investigated for studying the operational system effects of natural laminar flow aircraft. The assumed flow conditions affect the fuel efficiency of the FSW-NLF aircraft and are defined as follows: Optimum natural laminar flow refers to perfect operating conditions at the wing (approximately $15 \%$ of the aircraft's surface are laminar). Reduced natural laminar flow assumes a transition location reduced by $50 \%$ (approximately $7.5 \%$ of the aircraft's surface are laminar) e.g., due to a contamination of the wings. With turbulent boundary layer, no laminar flow at the wings is assumed at all. Variation of the turn-around time refers to the FSW-NLF aircraft configuration only, whereas the turn-around time of the reference fleet type is fixed to $30 \mathrm{~min}$ for all scenarios. All fixed and variable parameters for network design are listed in table 3 and table 4 . 
Table 3. Fixed network design assumptions.

\begin{tabular}{lc} 
Parameter & Assumption \\
\hline \hline Network strategy & point-to-point \\
Airports & 38 \\
Candidate markets & 76 \\
Candidate flights & 105 \\
Planning horizon & single day \\
\hline Fleet size / fleet type & max 100 \\
Seat capacity & 150 \\
Turn-around baseline fleet & $30 \mathrm{~min}$ \\
\hline
\end{tabular}

Table 4. Fuel price scenarios and parameter variation for airline network design.

\begin{tabular}{lccc} 
Parameter & Scenario-1 & Scenario-2 & Scenario-3 \\
\hline \hline Fuel price $[$ USD/barrel] & 75 & 100 & 150 \\
\hline Flow condition FSW-NLF wing & turbulent, reduced laminar flow \\
& \multicolumn{2}{c}{ and optimum laminar flow } \\
Turn-around FSW-NLF aircraft $[\mathrm{min}]$ & \multicolumn{3}{c}{$30,35,40$}
\end{tabular}

For each scenario and parameter variation, a profit-maximizing airline network is designed with the network optimization model introduced in the previous section. In the network design process, the optimizer decides about flights to be included in the network (out of the candidate flight list), and about the operational fleet. The resulting networks are evaluated with respect to network share of both fleet types and changes in global network profit and fuel consumption due to the introduction of FSW-NLF fleet. Network changes are compared to a reference network designed for each scenario with the baseline fleet only.

All scenarios include the same set of airports and candidate markets used as input for network design. Airports and candidate flights are selected from an existing flight schedule of a European low-cost carrier for a representative day in September 2010. Flights of the schedule are limited to those flown by the baseline fleet type (similar to A320). Note that in this study, the airline specific flights are used as a candidate flight list for network design and fleet assignment. Therefore, the network design problem becomes mainly a fleet assignment problem. However, in future work, the candidate flight list will be constructed from scratch resulting in generic airline networks designed for varying demand scenarios.

Market demand and ticket fares are retrieved from a global market intelligence database. ${ }^{16}$ The database contains aggregated demand and fare data on a true origin-destination basis, including passenger bookings from so-called market data information tapes. In this study, airline specific fares and market shares of the representative airline are used as input for network design and fleet assignment.

Operating costs for all candidate flights are calculated with a standard direct operating cost (DOC) method. ${ }^{17}$ This method is based on the ATA-67 standard method for DOC calculations. For the low-cost business case study, operating costs (excluding fuel and capital costs) are downscaled with a factor of 0.7 .

All networks designed for the different air transportation scenarios are restricted to the same size (within a $10 \%$ bound) in terms of available seat kilometers flown. This allows for better comparison of the performance of the different networks.

\section{B. Calculation of Mission Fuel Rates}

In order to consider the payload-range characteristic and the flight performance of the baseline and the FSW-NLF aircraft, flight mission simulations were performed in order to calculate mission fuel rates and mission times before network design. The goal was to determine required mission fuel and mission time for different stage lengths, cruise Mach numbers and initial cruise altitudes (ICA). This was done by applying parts of the CATS simulation chain, ${ }^{18,19}$ which was developed at DLR in order to assess the climate impact 
of aircraft for different mission profiles and aircraft designs.

The mission stage length of the simulations was varied between 250 and $5750 \mathrm{~km}$ and included simulations for the baseline fleet and the FSW-NLF fleet with the three defined flow conditions. The resulting mission fuel and block time regressions over flight distance serve as input for network design. The assumed load factor for mission simulation was 0.8. Natural laminar flow was assumed only during cruise. During cruise the $c_{L}$ from ICA conditions is kept fixed, leading to a continuous climb cruise. In order to select an appropriate trajectory for the respective mission length, some constraints were taken into account. On very short missions both configurations will not reach their design ICA. To determine typical cruise flight levels, original flight data from the Eurocontrol Central Flow Management Unit (CFMU) ${ }^{\mathrm{b}}$ were consulted. The data showed a wide spread of cruise flight levels on missions between 250 and $750 \mathrm{~km}$. For this reason, average cruise flight levels for these missions were computed and applied for the study (see figure 5). On missions longer than $1000 \mathrm{~km}$, no further altitude restriction exists. Beside the ICA constraints for missions with short stage lengths, further boundary conditions were set for cruise flight. It was paid attention that the cruise Mach number is 0.78 and the lift coefficient $c_{L}$ is kept within a range between 0.5 and 0.55 . Especially for the FSW-NLF configuration, a deviation from the $c_{L}$-range would lead to a reduced laminar flow area with declined flight performance due to off-design conditions. In case the allowed $c_{L}$-range is violated at any point of the mission, the next lower ICA is chosen to reduce the $c_{L}$ at cruise. Figure 5 gives an overview about the chosen ICAs. It can be noticed that from $2,500 \mathrm{~km}$ on, both aircraft operate on different ICAs. This is due to the fact that the FSW-NLF configuration with its better aerodynamical performance is able to fly longer within the defined $c_{L}$-range.

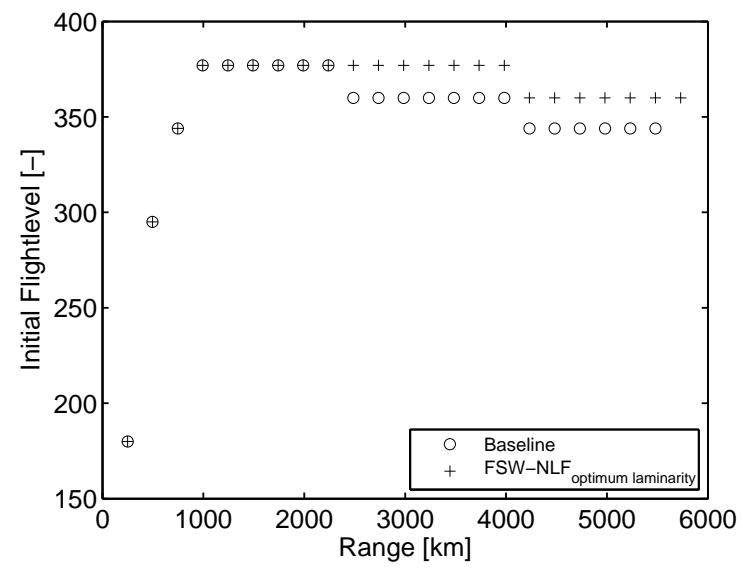

Figure 5. Chosen initial cruise flight levels for the FSW-NLF and the baseline aircraft.

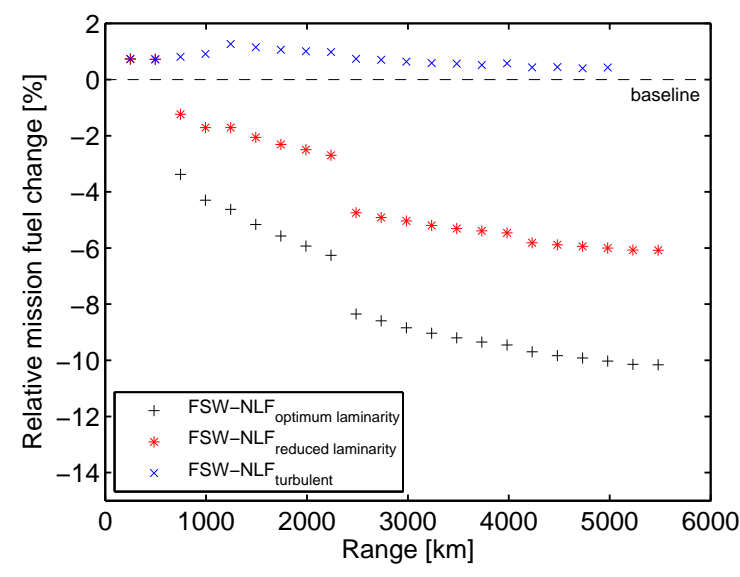

Figure 6. Mission fuel change due to use of the FSWNLF aircraft.

Figure 6 shows the relative change in mission fuel between the FSW-NLF configuration operating with the three different laminar flow conditions and the baseline configuration. Looking at the first and second mission, higher fuel consumption for all flow conditions can be observed. On these missions, off-design conditions provoke the loss of laminar flow. For stage lengths longer than $750 \mathrm{~km}$, the FSW-NLF configuration with optimum laminar flow shows fuel burn improvements between 3 and $10 \%$. Even with reduced laminar flow, the FSW-NLF configuration provides a fuel consumption advantage of up to $6 \%$. The discontinuities in the fuel saving curves at a range of 2, 500 km are the result of the different ICAs of the two aircraft. Flying at higher altitudes goes along with a reduced drag, offering some further advantage for the FSW-NLF. In the event of a complete loss of natural laminar flow, the mission fuel on short ranges is approximately $1 \%$ higher compared to the baseline due to the higher OEW.

\section{Network Results}

The results of the designed networks are listed in the tables 5-7. table 5 shows the baseline network results for all three fuel price scenarios. The table reveals a fleet size of 19 aircraft of the baseline fleet type

\footnotetext{
bEurocontrol CFMU data internally provided by the DLR project "Weather \& Flying"
} 
required for network operation. Network size is fixed for all scenarios, therefore, approximately the same flights are flown in each scenario. Global network profit declines from 810,081 USD to 425, 383 USD with increasing fuel prices. This has been expected as increased fuel price costs are assumed to be internalized by the airline but not passed on to customer fares. Further, the table shows the break-even load factor (BELF) for the different scenarios, which is an important airline measure for network profitability apart from absolute profits. The BELF indicates the airline network load factor required to break-even, i.e., to cover all direct operating costs. Here, BELF is simply calculated by dividing network unit costs (costs per available seat kilometer) by network yield (revenue per passenger seat kilometer). The lower the BELF, the higher the potential profit margin. In table 5, BELF rises from 49.8 up to 63.3 percent. That is, for the 150 USD fuel price scenario, the airline has to achieve a network load factor of at least $63.3 \%$ in order to break even as unit costs increased but yields are constant. This means, profit margins are much lower compared to the 75 USD fuel price scenario assuming a constant network load factor (e.g. $80 \%$ ) achieved by the airline. Finally, global network fuel consumption is shown to be 607 metric tons per day for all scenarios as network size is constant, i.e., no flights are added or deleted. A network visualization ${ }^{\mathrm{c}}$ of a reference network designed with the baseline for the medium fuel price scenario is shown in figure 7 .

Table 5. Network performance with baseline fleet.

\begin{tabular}{lccc}
\hline \hline Baseline fleet & Scenario-1 & Scenario-2 & Scenario-3 \\
Fuel price [USD/barrel] & 75 & 100 & 150 \\
\hline \hline Baseline fleet size & 19 & 19 & 19 \\
Profit [USD] & 810081 & 690848 & 452383 \\
BELF [\%] & 49.8 & 54.3 & 63.3 \\
Fuel [t] & 607 & 607 & 607 \\
\hline
\end{tabular}

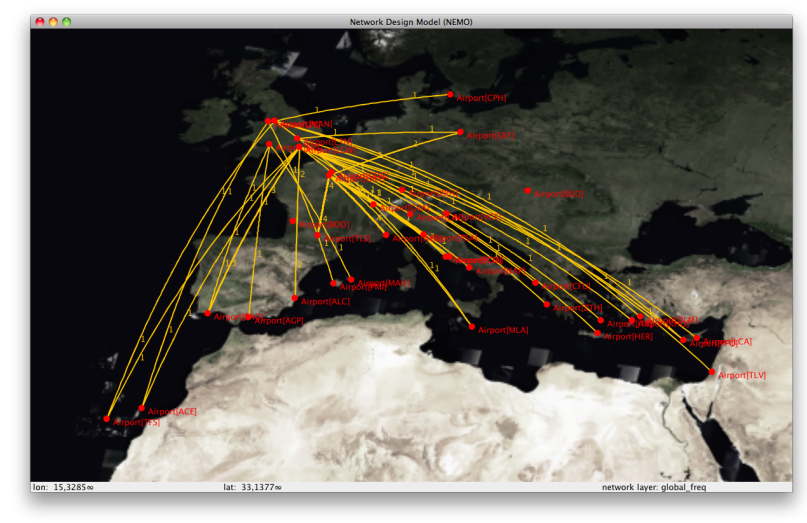

Figure 7. Reference network designed with baseline fleet for 100 USD/barrel fuel price scenario.

Table 6 shows network results for the same fuel price scenarios but now designed with the baseline and the FSW-NLF fleet available as an additional fleet option. That is, the model is free to select its operational fleet from both aircraft types. As the fleet size is unrestricted (here set to a theoretical maximum of 100 aircraft), fleet decisions mainly depend on fleet performance characteristics such as fuel efficiency and turn-around time. In table 6, turn-around time is assumed to be equal $(30 \mathrm{~min})$ for both the baseline and the FSW-NLF fleet. However, network results in table 6 are divided into three rows assuming different fuel efficiencies of the FSW-NLF aircraft dependent on the flow condition at the wings (turbulent, reduced and optimum laminar flow). Clearly, fuel efficiency of the FSW-NLF aircraft affects fleet composition and network performance. Profit, BELF and fuel changes in table 6 refer to network results operated by the baseline fleet only as shown in table 5. The first row assumed the FSW-NLF aircraft to be operated in conventional turbulent flow. This is a worst case scenario for the FSW-NLF fleet. There are no benefits compared to the baseline fleet as network share of the FSW-NLF fleet is 0 for all fuel price scenarios. That is, under turbulent flow conditions

${ }^{\mathrm{c}}$ Map data in this paper is provided by (C) Unearthed Outdoors, LLC. 
the FSW-NLF aircraft is not operated at all but the baseline aircraft is used instead. Here, network share is shown by the number of seat kilometers (ASK) flown by the FSW-NLF fleet relative to the total number of seat kilometers available in the network.

The second row in table 6 assumes a FSW-NLF fleet with reduced laminar flow on the wing surface, corresponding to an aircraft surface of about $7.5 \%$. Here, network share of the FSW-NLF fleet is between 86 and 90 percent dependent on fuel price scenario. That is, network share of the FSW-NLF fleet exceeds the network share of the baseline fleet, resulting in total profit increases of $0.8 \%$ for the 75 USD fuel price scenario up to $3.1 \%$ for the 150 USD fuel price scenario. Profit increases are due to the increased fuel efficiency of the FSW-NLF aircraft compared to the baseline aircraft. Accordingly, the BELF declines from 0.5 to 0.8 percent, which increases the potential airline profit margin. Global network fuel savings due to the introduction of the FSW-NLF fleet are $2.2 \%$ for all scenarios. Fuel savings are constant among the fuel price scenarios, which indicates the maximum fuel efficiency of the FSW-NLF fleet is exploited in each scenario.

Lastly, the third row assumes optimal natural laminar flow conditions for the FSW-NLF aircraft, corresponding to an aircraft surface with laminar flow of about $15 \%$. Results show FSW-NLF network share to be $100 \%$, i.e., the baseline fleet is operated no more. Network profits increase from 3.4 up to 12.7 percent compared to the same network operated with the baseline fleet only. BELF declines from 2.1 up to 3.5 percent, and fuel savings are 8.2 percent compared to the baseline fleet. Network visualizations with varying NLF-performance are given in figure 8. The figures show the network share flown by the baseline fleet and the FSW-NLF fleet for each scenario.

Table 6. Network performance with baseline and FSW-NLF fleet.

\begin{tabular}{lccc}
\hline \hline Baseline + FSW-NLF fleet & Scenario-1 & Scenario-2 & Scenario-3 \\
Fuel price [USD/barrel] & 75 & 100 & 150 \\
\hline \hline ASK share FSW-NLF turbulent [\%] & 0 & 0 & 0 \\
\hline ASK share FSW-NLF reduced [\%] & 86 & 90 & 90 \\
$\Delta$ Profit [\%] & +0.8 & +1.3 & +3.1 \\
$\Delta$ BELF [\%] & -0.5 & -0.6 & -0.8 \\
$\Delta$ Fuel [\%] & -2.2 & -2.2 & -2.2 \\
\hline ASK share FSW-NLF optimum [\%] & 100 & 100 & 100 \\
$\Delta$ Profit [\%] & +3.4 & +5.5 & +12.7 \\
$\Delta$ BELF [\%] & -2.1 & -2.6 & -3.5 \\
$\Delta$ Fuel [\%] & -8.2 & -8.2 & -8.2 \\
\hline
\end{tabular}

Table 7. Turn-around impact for 100USD/barrel fuel price scenario.

\begin{tabular}{lccc}
\hline \hline Baseline + FSW-NLF fleet & Scenario-1 & Scenario-2 & Scenario-3 \\
FSW-NLF turn-around [min] & 30 & 35 & 40 \\
\hline \hline ASK share FSW-NLF reduced [\%] & 90 & 70 & 50 \\
$\Delta$ Profit [\%] & +1.3 & +1.0 & +0.8 \\
$\Delta$ BELF [\%] & -0.6 & -0.5 & -0.4 \\
$\Delta$ Fuel [\%] & -2.2 & -1.6 & -1.3 \\
\hline ASK share FSW-NLF optimum [\%] & 100 & 70 & 50 \\
$\Delta$ Profit [\%] & +5.5 & +4.1 & +3.1 \\
$\Delta$ BELF [\%] & -2.6 & -2.0 & -1.5 \\
$\Delta$ Fuel [\%] & -8.2 & -6.0 & -4.7 \\
\hline
\end{tabular}

All results in table 6 with an assumed equal turn-around represent a case where fleet decisions depend on fuel efficiency only. In contrast, table 7 shows network results assuming different turn-around times for the FSW-NLF aircraft compared to the baseline aircraft. Turn-around time of the baseline aircraft is assumed to be fixed to $30 \mathrm{~min}$. The first scenario shows results based on equal turn-around assumption as 
already presented in table 6 . The second scenario assumes a five minute turn-around time drawback of the FSW-NLF aircraft over the baseline aircraft. As can be seen in table 6, a five minute turn-around drawback e.g., due to increased maintenance effort for cleaning of the wings after each flight, results in significant changes in network results. Basically, network share of the FSW-NLF fleet with reduced natural laminar flow assumption decreases by 20 percent from $90 \%$ with equal turn-around to $70 \%$. Accordingly, profits and savings in fuel consumption decrease. With $10 \mathrm{~min}$ turn-around drawback of the FSW-NLF fleet, network share goes even further down to 50 percent compared to a 90 percent share with 30 min turn-around. Similar results are obtained for the FSW-NLF fleet with optimal natural laminar flow condition. Results indicate a significant impact of network effects in terms of fleet utilization due to an increased turn-around time of the FSW-NLF fleet. That is, a five minute turn-around time increase for each flight propagates through the network and affects network results significantly over the day. The full potential in fuel efficiency of the FSW-NLF fleet cannot be fully exploited as fleet utilization goes down. The respective network shares flown by the baseline fleet and the FSW-NLF fleet for different turn-around scenarios are visualized in figure 9 .

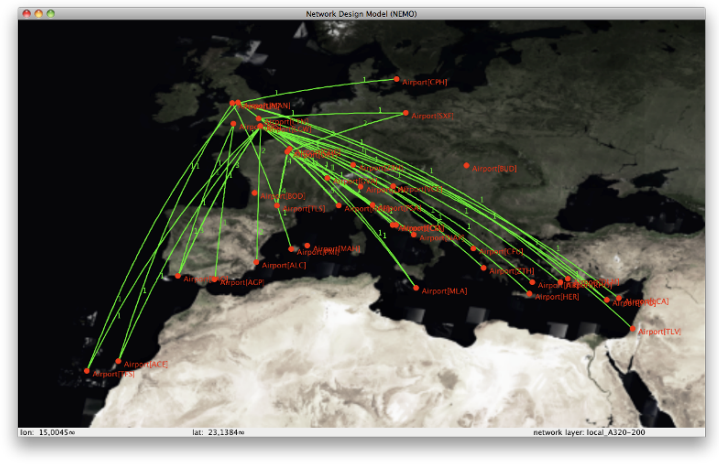

(a) Scenario FSW-NLF turbulent: Baseline fleet.

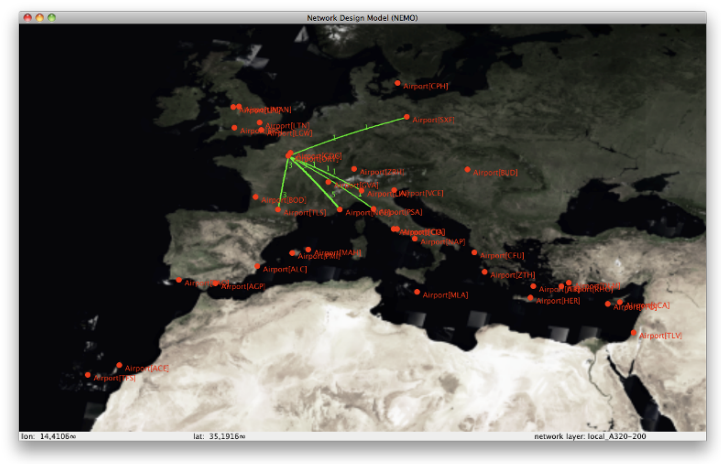

(c) Scenario FSW-NLF reduced NLF: Baseline fleet.

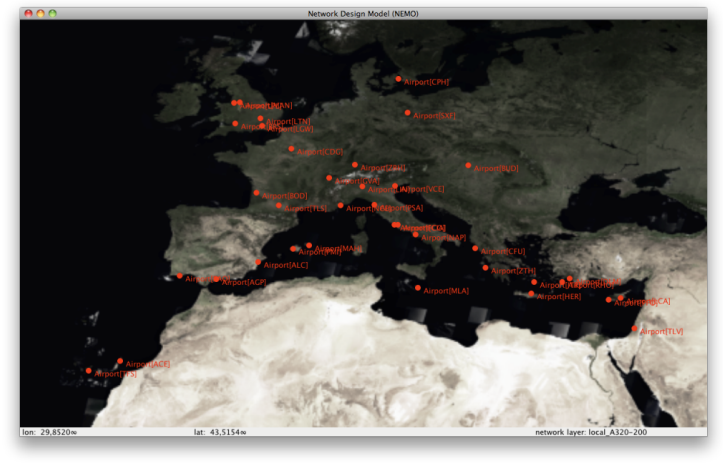

(e) Scenario FSW-NLF optimum NLF: Baseline fleet.

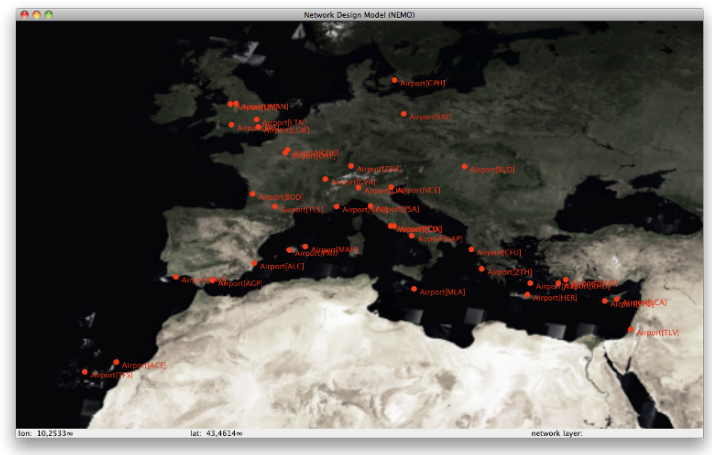

(b) Scenario FSW-NLF turbulent: FSW-NLF fleet.

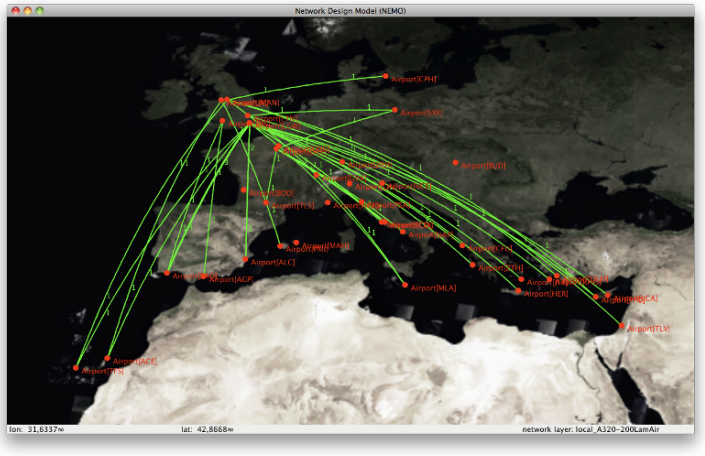

(d) Scenario FSW-NLF reduced NLF: FSW-NLF fleet.

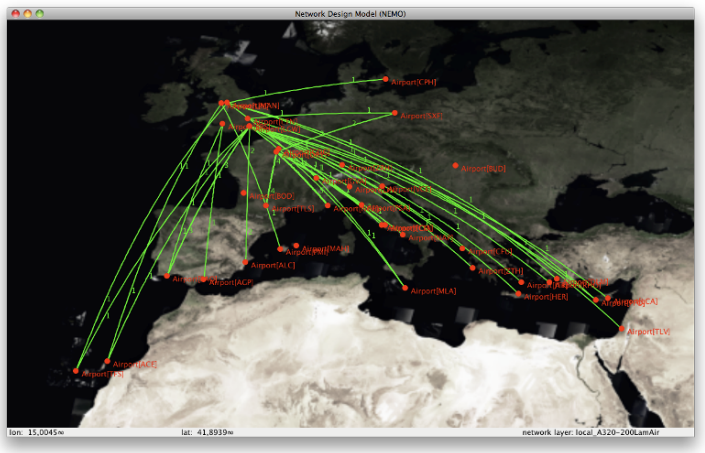

(f) Scenario FSW-NLF optimum: FSW-NLF fleet.

Figure 8. Network visualization for NLF conditions scenarios. Assumptions: 100USD/barrel fuel price and equal turn-around time. 


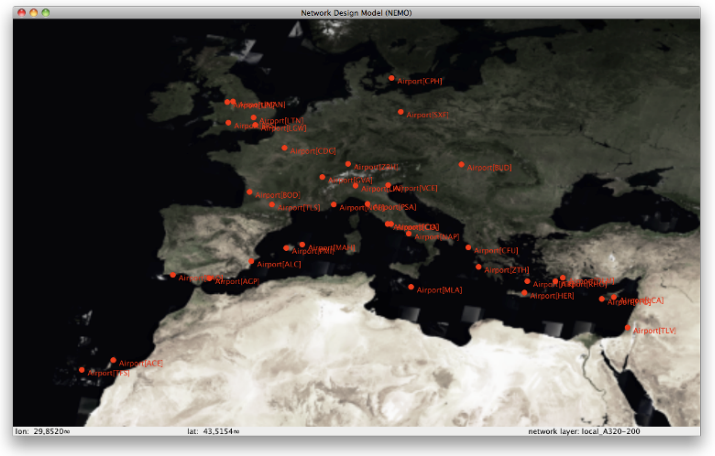

(a) 30min TA-Scenario for FSW-NLF: Baseline fleet.

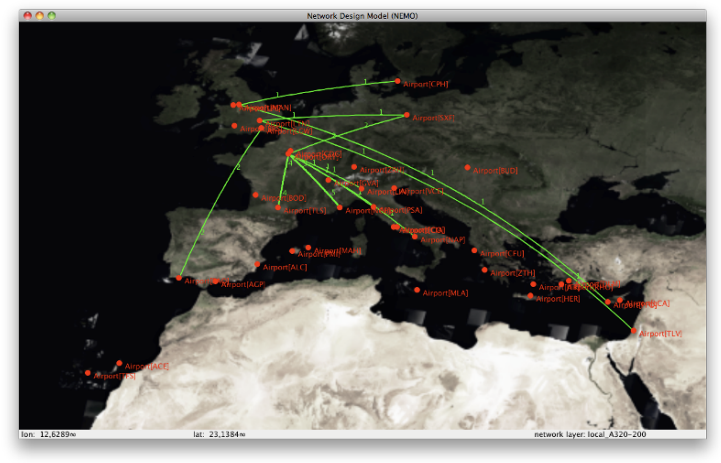

(c) 35min TA-Scenario for FSW-NLF: Baseline fleet.

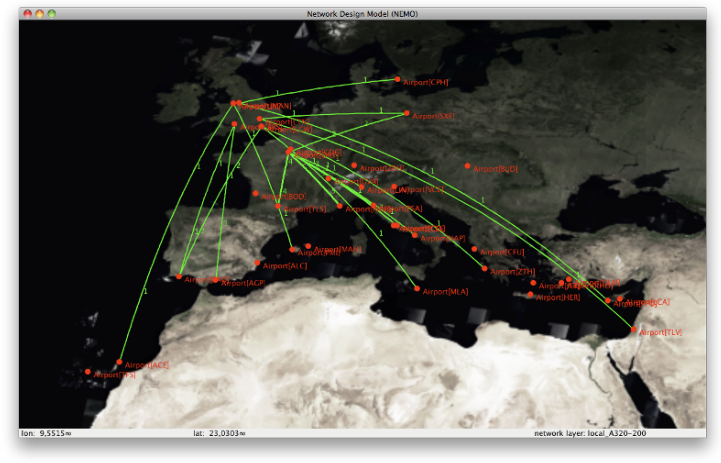

(e) 40min TA-Scenario for FSW-NLF: Baseline fleet.

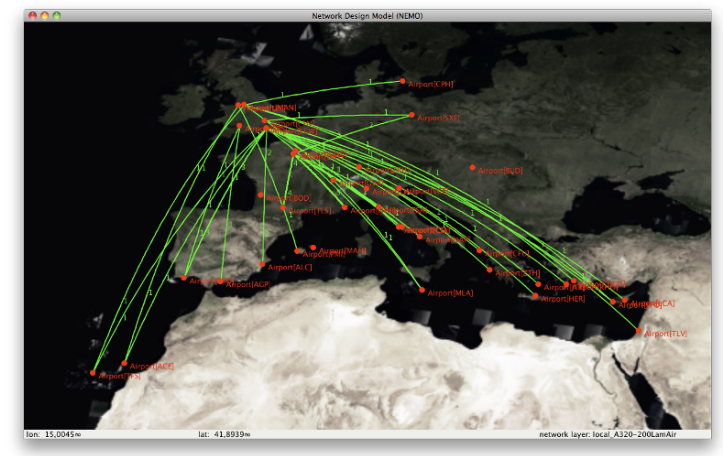

(b) 30min TA-Scenario for FSW-NLF: FSW-NLF fleet.

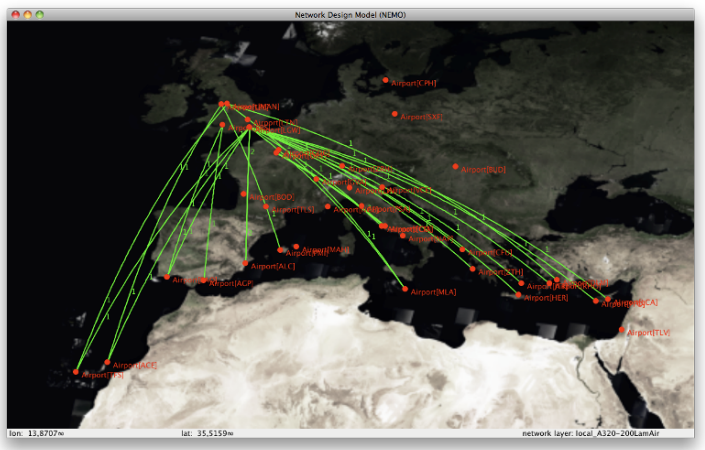

(d) 35min TA-Scenario for FSW-NLF: FSW-NLF fleet.

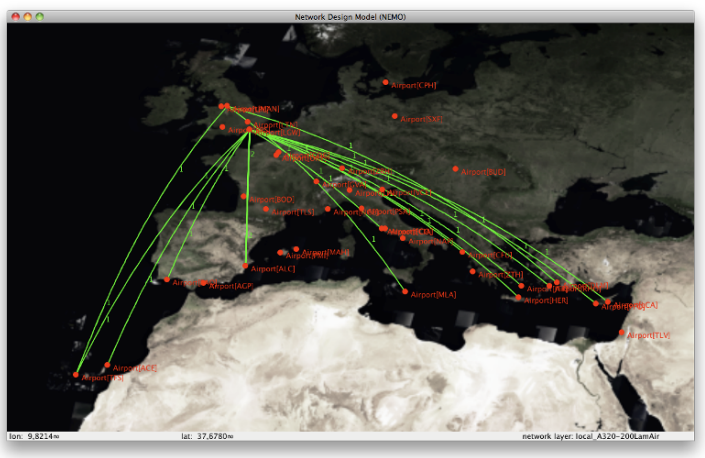

(f) 40min TA-Scenario for FSW-NLF: FSW-NLF fleet.

Figure 9. Turn-around (TA) scenarios for FSW-NLF fleet with constant turn-around time of 30min for baseline fleet. Assumptions: 100USD/barrel fuel price and optimum NLF conditions.

\section{Conclusion}

This paper showed the evaluation of a future aircraft concept equipped with natural laminar flow technology leading to higher fuel efficiency in direct comparison to existing state-of-the-art short-to-medium haul aircraft. Evaluation was based on network modeling from a single airline perspective in contrast to cost-benefit analysis applied to a single standard flight mission. The benefit of a network based evaluation approach is the capturing of network effects such as changes in fleet turn-around time. Results show that network effects are significant from an airline perspective as they can propagate through a network over time, leading to significant impacts on network profitability. All results were carried out with an integrated network design and fleet assignment model. The model was used to design networks for different air transportation scenarios and to select the most appropriate fleet composition dependent on the scenario. In this study, an 
existing daily flight schedule served as a candidate flight list for network design and fleet assignment. In addition to a standard fleet assignment formulation, the model considers capital costs of the operated fleet in order to be able to study fleet utilization effects such as those induced by changes in fleet turn-around time.

Network results showed a significant impact of the assumed natural laminar flow condition (turbulent, reduced, optimum) of the future concept on fleet composition and network profitability, independent of the fuel price scenario. Under turbulent flow conditions, the future aircraft concept is not operated at all. However, with reduced and optimum NLF areas, the increased fuel efficiency leads to increases in network profit with significant network share of the FSW-NLF fleet. Under the given assumptions for network design and technology evaluation, network fuel savings are potentially in the range of 2 up to 8 percent due to introduction of FSW-NLF aircraft into the operational fleet. Further, results revealed turn-around time to be a main decision driver for introduction of FSW-NLF aircraft into the operational fleet. According to results, 5-10 minutes turn-around time drawback of the future concept in comparison to the baseline fleet leads to significant losses in network share and network contribution of the future concept. This confirms the importance of network effects to be considered in technology evaluation of future concepts.

This work also revealed some limitations of using an existing flight schedule for technology evaluation of future air transportation system concepts. For example, a $45 \mathrm{~min}$ turn-around time scenario could not be evaluated as the fixed flight schedule is optimized for lower turn-around times. In addition, today's airline schedules are optimized for the current business environment e.g., in terms of passenger demand and fleet technology. Future work focuses on technology evaluation in a hub-and-spoke business case and on the evaluation of different demand scenarios using a more flexible network design approach. The future approach will be based on a generic candidate flight list used for network design instead of an existing airline schedule.

\section{Acknowledgments}

The authors would like to express their gratitude for the allocation of the simulation chain developed in the framework of the DLR CATS project ${ }^{18,19}$ in order to compute the mission fuel rates and mission times in this paper.

\section{References}

${ }^{1} \mathrm{ICAO}$, ICAO Environmental Report 2007, Montreal, Quebec, Canada, 2010, URL: http://www.icao.int/env/ pubs/env_report_07.pdf.

${ }^{2}$ Joslin, R.D., Overview of Laminar Flow Control, NASA/TP-1998-208705, Langley Research Center, Hampton, Virginia, 1998.

${ }^{3}$ Werner-Westphal, C., Heinze, W., and Horst, P., Multidisciplinary Integrated Preliminary Design Applied to Future Green Aircraft Configurations, 45th AIAA Aerospace Sciences Meeting and Exhibit, 8 - 11 January 2007, Reno, Nevada, 2007.

${ }^{4}$ Werner-Westphal, C., Heinze, W., and Horst, P., Multidisciplinary Integrated Preliminary Design Applied to Unconventional Aircraft Configuration, Journal of Aircraft, Vol. 45, No. 2, 2008, pp. 581-590.

${ }^{5}$ Schrauf, G., Status and perspectives of laminar flow, Aeronautical Journal, Vol. 109, No. 1102, 2005, pp. 639-644.

${ }^{6}$ Holmes, B.J., and Obara, C.J., Observation and Implications of Natural Laminar Flow on Practical Airplane Surfaces, Journal of Aircraft, Vol. 20, No. 12, 1983, pp. 993-1006.

${ }^{7}$ Rossow, C.-C., Aerodynamics - A discipline swept away?, Aeronautical Journal, Vol. 114, No. 1160, 2010, pp. 599-609.

${ }^{8}$ Horstmann, K.-H., and Streit, T., Aerodynamic Wing Design for Transport Aircraft - Today, Hermann Schlichting 100 Years, Springer-Verlag, Berlin, Heidelberg, 2009, pp. 130144.

${ }^{9}$ Redeker, G., and Wichmann, G., Forward Sweep - A Favorable Concept for a Laminar Flow Wing, Journal of Aircraft, Vol. 28, No. 2, 1991, pp. 97-103.
${ }^{10}$ Weisshaar, T.A., Aeroelastic Tailoring of Forward Swept Composite Wings, Journal of Aircraft, Vol. 18, No. 8, 1981, pp. 669-676.

${ }^{11}$ Librescu, L., and Khdeir, A.A., Aeroelastic Divergence of Swept-Forward Composite Wings Including Warping Restraint Effect, AIAA Journal, Vol. 26, No. 11, 1988, pp. 13731377.

${ }^{12}$ Monner, H.P., Kintscher, M., Lorkowski, T. and Storm, S., Design of a Smart Droop Nose as Leading Edge High Lift System for Transportation Aircraft, 50th AIAA/ASME/ASCE/AHS/ASC Structures, Structural Dynamics, and Materials Conference, 4-7 May 2009, Palm Springs, California, 2009, AIAA 2009-2128.

${ }^{13}$ Gopalan, R., and Talluri, K.T., Mathematical Models in Airline Schedule planning. A Survey, Annals of Operations Research, Vol. 76, 1998, pp. 155-185.

${ }^{14}$ Hane, C.A., and Barnhart, C., et al., The Fleet Assignment Problem - Solving a Large-Scale Integer Program, Mathematical Programming, Vol. 70, No. 2, 1998, pp. 211-232.

${ }^{15}$ Lohatepanont, M., and Barnhart, C., Airline Schedule Planning: Integrated Models and Algorithms for Schedule Design and Fleet Assignment, Transportation Science, Vol. 38, No. 1, 2004, pp. 19-32.

${ }^{16}$ Sabre. Airport Data Intelligence, Southlake, Texas, 2011, URL: http://www.sabreairlinesolutions.com/home/ products_services/airports.

${ }^{17}$ Liebeck, R.H., et al. Advanced Subsonic Airplane design and Economic Studies, NASA CR-195443, Langley Research Center, Hampton, Virginia, 1998. 
${ }^{18}$ Koch, A., Nagel, B., Gollnick, V., Dahlmann, K., Grewe, V., Kärcher, B., and Schumann, U. Integrated analysis and design environment for a climate compatible air transport system, 9th AIAA Aviation Technology, Integration, and Operations Conference, 21-23 September 2009, Hilton Head, South Carolina, 2009, AIAA 2009-7050.
${ }^{19}$ Koch, A., Dahlmann, K., Linke, F., Grewe, V., Plohr, M., Gollnick, V., Schumann, U., and Lührs, B., Climate impact assessment of varying cruise flight altitudes applying the CATS simulation approach, CEAS 3rd Air and Space Conference, 24-28 October, Venice, Italy, 2011. 